$>$ En France, la donnée génétique n'est pas accessible au travers du droit de la propriété. Elle est assimilée à un élément du corps humain à protéger, et non un bien à valoriser. Pourtant, dans notre monde extrêmement concurrentiel, il est indispensable que la France trouve des solutions lui permettant de valoriser, scientifiquement et économiquement, les données génétiques de façon plus efficiente. Une possibilité serait de définir la donnée génétique comme une information brute dépourvue de valeur d'usage. Ce sera donc le choix d'une valeur d'usage (clinique, scientifique, économique, etc.) obtenue suite à différents processus d'analyse et de traitement significatifs, qui la transformeront en une information utile. Dans ce cas et sous certaines conditions, cette information peut être alors considérée par le droit de la propriété intellectuelle comme une création originale et ainsi être valorisée économiquement, tout en maintenant le même niveau de protection actuel sur les données génétiques. La France a donc le choix entre changer la loi sur la protection des données génétiques, en se rapprochant de l'approche nord-américaine, considérant ces données comme un capital que chacun peut faire fructifier ou vendre, ou établir une distinction franche entre la donnée génétique et l'information. <

Le choix d'une économie numérique mène inéluctablement les sociétés à se réorganiser autour de l'exploitation des données. La «donnée », au sens d'une information brute dépourvue de valeur d'usage clairement définie, possède désormais une valeur d'échange dont l'importance dépend de sa nature. Sur ce gigantesque marché mondial, plus la donnée est relative à la santé d'un individu, plus la valeur d'échange est grande.

Vignette (Photo (c) Inserm - Claudine Lemercier).

\title{
La propriété des données génétiques
} De la donnée

\section{à I'information}

Henri-Corto Stoeklé ${ }^{1,2 *}$, Ninon Forster ${ }^{3 *}$,

Mauro Turrini ${ }^{4}$, Philippe Charlier ${ }^{5,6}$, Christian Hervé ${ }^{7}$, Jean-François Deleuze ${ }^{2,8,9}$,Guillaume Vogt ${ }^{1,2,10}$

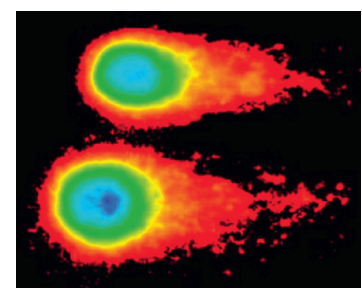

La donnée génétique est alors l'une de celles qui, pour l'instant, possède une valeur d'échange qui est particulièrement importante lorsqu'elle est associée à des processus biologiques qui ont, ou peuvent avoir, une valeur diagnostique ou pharmacologique. La donnée génétique, en particulier de santé, devient un capital que l'on peut faire fructifier ou vendre. Elle tend à être considérée comme un bien valorisable économiquement par chacun d'entre nous. Mais cette idée, largement acceptée aux États-Unis, ne l'est pas forcément dans I'Union européenne, ou du moins dans certains de ses États membres, comme la France. En France, une donnée génétique n'est pas considérée comme un bien, et encore moins comme une entité valorisable économiquement par chacun d'entre nous. Elle peut l'être, mais collectivement, suite, en général, à des projets de recherche académique respectant un cadre réglementaire strict qui interdit, en particulier, la rémunération du «donneur», qui n'est donc pas considéré comme un «proprié-
${ }^{1}$ Laboratoire de Génétique Humaine Négligée, CNRGH-CEA, Évry, France.

${ }^{2}$ Centre National de Recherche en Génomique Humaine (CNRGH), Direction de la recherche fondamentale, CEA, Institut de biologie François Jacob, Université Paris Saclay, Évry, France. ${ }^{3}$ Centre de Droit Européen, Université Paris II PanthéonAssas, Paris, France.

${ }^{4}$ Université de Nantes - Maison des Sciences de l'Homme (MSH), Nantes, France.

${ }^{5}$ Département de la Recherche et de l'Enseignement, Musée du Quai Branly-Jacques Chirac, 222, rue de l'Université, 75007 Paris, France.

${ }^{6}$ UVSQ (Laboratoire DANTE - EA 4498), 2, avenue de la source de la Bièvre, 78180 Montigny-leBretonneux, France.

${ }^{7}$ Académie Internationale Éthique, Médecine et Politiques Publiques, Université Paris Descartes, Paris, France. ${ }^{8}$ LaBex GenMed, Fondation Jean Dausset, Paris, France.

${ }^{9}$ Centre d'études du polymorphisme humain (CEPH), Fondation Jean Dausset, Paris, France.

${ }^{10}$ Laboratoire de Génétique Humaine Négligée, Inserm, Université Paris Descartes, Paris, France.

*Ces auteurs ont contribué également

guillaume.vogt@inserm.fr 
taire ». La donnée génétique n'est pas saisissable à travers le droit de la propriété, comme éventuellement pourrait s'en rapprocher le droit aux États-Unis, qui possèdent évidemment un régime juridique propre et distinct du nôtre sur ces questions. En France, la donnée génétique n’est en effet pas dissociable juridiquement de la personne et du cadre juridique qui organise sa protection vis-à-vis des autres et vis-à-vis d'elle-même. Elle est un élément du corps humain à protéger, et non un bien à valoriser. Mais, dans un monde extrêmement concurrentiel, ce cadre juridique suscite différentes interrogations en raison des obstacles qu'il impose, en France, à la valorisation efficiente des données génétiques, tant économique que scientifique, ne fut-elle que collective.

\section{La valorisation économique des données génétiques aux États-Unis}

Aujourd'hui, un citoyen américain, majeur, a la possibilité d'avoir accès à ce que la société considère comme «ses » données génétiques [1]. Surtout, une entreprise privée peut être en droit de produire ces données génétiques, à partir d'un échantillon biologique que l'individu lui aura préalablement transmis via l'achat d'un service, mais aussi de les stocker afin de les valoriser de différentes façons, dont économiquement, auprès de tiers, sous couvert d'un contrat commercial et/ ou d'un consentement [2]. Cela peut être réalisé avec ou sans prescription médicale selon l'entreprise privée en question. Par exemple, l'accès au test génétique de Myriad Genetics demande une prescription médicale, contrairement au test de 23 andMe. La raison de cela réside dans la nature même du test proposé : le premier est un dispositif médical agréé par la Food and drug administration (FDA), l'autre uniquement en partie, et cela depuis peu [3, 4].

Depuis les années 1990, les États-Unis ont vu apparaître ces nouvelles entreprises privées de biotechnologie, appelées plus communément «biotechs », spécialisées dans la production et le stockage de données génétiques [5]. Myriad Genetics et 23andMe sont loin d'être les seules, mais elles restent les plus célèbres mondialement. Elles le sont d'une part en raison de leur réussite commerciale avérée et, d'autre part, en raison des différentes réactions que leurs tests génétiques et leur modèle économique ont suscité dans les médias ou la presse spécialisée aux États-Unis, mais aussi en Europe, et plus particulièrement en France [6]. Le modèle du « direct-to-consumer » a fait et continu de faire débat, en particulier sur les modalités d'accès, de stockage et d'usage des données génétiques que ces entreprises privées «produisent à la chaîne » [7].

Pour bien aborder ces questions, il est nécessaire avant tout de comprendre que la situation est devenue davantage complexe depuis le début des années 2000 pour différentes raisons qui vont au-delà de la génétique. En effet, outre évidemment la «révolution» du séquençage à haut débit, qui permet une production significativement plus importante de données génétiques, elle correspond aussi à l'avènement d'internet comme moyen ultime de communication et d'accès à l'information. Mais aujourd'hui, les moyens de communication usuels (ordinateur, smartphones, tablettes) sont devenus des moyens de production «spontanée » de données personnelles [8, 9]. Chaque individu peut alimenter en données de toute nature le réseau numérique auquel il est relié, contrôlé majoritairement par quelques entreprises privées américaines, appelées GAFA (Google, Amazon, Facebook, Apple). Ces dernières ont développé différentes techniques et pratiques leur permettant de collecter ces données, puis de les transformer en information de façon massive.

La production de données génétiques et leur transformation en information reste cependant centralisée [8]. Seules des biotechs (ainsi que certaines pharmas) ou des structures académiques sont actuellement en mesure de les produire, de les stocker, de les transformer et de les valoriser scientifiquement et/ou économiquement. Mais l'idée spécifique à certaines biotechs, comme 23 andMe, et non aux structures académiques, a été d'adapter le modèle économique des GAFA dans le domaine de la génétique. II s'agit alors de produire ces données afin de les transformer en information d'intérêt pour le consommateur du test génétique, et à les vendre, transformées ou non en information, à prix élevé à des tiers, en les stockant et les croisant avec d'autres données personnelles. Cela fut possible grâce à internet et un réseau numérique calqué sur celui des GAFA. C'est le principe d'un « marché biface » $[2,10,11]$.

Aux États-Unis, la donnée génétique semble donc être considérée comme un bien valorisable économiquement directement par une entreprise privée et un consommateur qui semblent chacun jouir de ce qui pourrait se rapprocher d'un droit de propriété à la Française vis-àvis des données génétiques. Au plan juridique français, la «propriété » est le droit de jouir et de disposer des choses de manière absolue. II se constitue de trois attributs: L'abusus (droit de disposer de son bien, qu'il s'agisse de la disposition juridique de son bien par l'aliénation - vente ou don - ou matérielle par la destruction), l'usus (droit d'user d'une chose, sans que l'on n'en perçoive les fruits) et le fructus (droit de disposer des fruits d'une chose). Mais cette idée de propriété reste, pour l'instant, extrêmement controversée au sein de l'Union européenne, plus particulièrement en France [12-14].

\section{La question de la propriété des données génétiques en France}

Cette controverse découle du fait que la donnée génétique est considérée par le droit français comme un élément du corps humain qui «appartient » à un «donneur », sans que pourtant il soit possible d'envisager cette protection sous l'angle du droit de propriété. Cela découle du principe de non-patrimonialité du corps humain $[15,16]^{1}$ : en France, le corps n'est pas un bien

${ }^{1}$ Articles, 16-1, alinéa 3, 16-5 et 16-6 du Code civil. 
dont une personne peut disposer et user, comme aux États-Unis. Ce principe, qui a fondé les principes de la gratuité et de l'anonymat des échanges des organes, des tissus et des gamètes humains [17], a été remis de plus en plus en question par la présence des collaborations entre partenaires publics et privés dans la pratique et, davantage encore, dans la recherche médicale de pointe [18].

Ce principe de non-patrimonialité a toutefois pour corollaire le principe de libre accès et de la mise en commun des bénéfices qui peuvent en être tirés, qu'ils soient intellectuels, médicaux ou économiques ${ }^{2}$. Ainsi, chaque personne peut disposer et user de ses propres données génétiques mais dans un espace bien précis, comme la recherche médicale, sans attendre aucun retour financier [19]. Le bénéfice direct sera collectif, et le bénéfice individuel, s'il y en a un, sera indirect. II s'agit donc bien d'un « don » ou d'un « partage » au sens d'une «distribution » consciente de biens matériels ou immatériels, sans réciprocité et obligation mutuelle, directes ou indirectes, nécessairement attendues [20]. Le «partage » renvoie également à un partage des bénéfices produits par la valorisation scientifique des données, en termes de contrôle à leur accès de la part des personnes qui les ont produites [20]. Ce «partage» doit néanmoins se faire dans le respect des règles du droit à la protection de la vie privée, notamment de la protection des données à caractère personnel ${ }^{3}$, à défaut de quoi celui qui utilise les données s'expose à des sanctions administratives et pénales ${ }^{4}$.

Le droit français a plutôt tendance à envisager la propriété des données génétiques sous l'angle des droits de la personnalité. Ces droits favorisent un régime juridique très protecteur vis-à-vis de la production et de l'usage des données dites sensibles, comme les données génétiques, fondé sur un respect de la confidentialité et un contrôle systématique et approfondi, par différentes institutions spécialisées, en particulier la Commission nationale de l'informatique et des libertés (CNIL). Cette idée est largement partagée par le droit de l'Union européenne. La donnée génétique bénéficie d'une protection particulière dans le cadre de la protection de données à caractère personnel ${ }^{5}$ et cette protection est considérée comme un droit fondamental ${ }^{6}$.

La distinction entre une personne et «ses » données génétiques est donc établie par le droit français et le droit de l'Union européenne. Les

${ }^{2}$ Avis $n^{\circ} 124$ du Comité consultatif national d'éthique pour les sciences de la vie et de la santé du 21 janvier 2016, Réflexion éthique sur l'évolution des tests génétiques liés au séquençage de l'ADN humain à très haut débit.

${ }^{3}$ Loi n²016-1321 du 7 octobre 2016, pour une République numérique, portant révision de la loi du 6 janvier 1978 relative à l'informatique, aux fichiers et aux libertés. Les données génétiques ne sont pas expressément visées par la loi, mais entrent dans la définition des «données à caractère personnel » exposée à l'article 2. Les données génétiques entrent dans le cadre des données sensibles prévues à la section 2 du chapitre 2 de la loi, à l'article 8, alinéa 1 ». Pour ces données, le principe est celui de l'interdiction de traitement et de collecte, tempéré par un certain nombre d'exceptions (article 7) qui encadrent strictement l'exploitation des données génétiques.

${ }^{4}$ Par exemple, TGI, Marseille, 7 juin 2007, $6^{\text {e }}$ ch. corr.

${ }^{5}$ Règlement (UE) n²016/679 du Parlement européen et du Conseil, du 27 avril 2016, relatif à la protection des personnes physiques à l'égard du traitement des données à caractère personnel et à la libre circulation de ces données, et abrogeant la directive 95/46/C $\varepsilon$, J0, L 119 du 4 mai 2016, pp. 1 à 88 articles 4 , alinéas 1,9 et 13 .

${ }^{6}$ Article 8 de la Charte des droits fondamentaux de l'Union européenne. Pour un rappel de l'évolution de la protection des données personnelles, voir annexe de l'avis $n^{\circ} 124$ du Comité consultatif national d'éthique pour les sciences de la vie et de la santé du 21 janvier 2016, Réflexion éthique sur l'évolution des tests génétiques liés au séquençage de l'ADN humain à très haut débit. données génétiques appartiennent à la personne dont elles sont issues, mais comme élément constitutif de son corps à protéger et non comme un bien à valoriser économiquement. Il existe donc bien une distinction entre la personne et ses données génétiques, mais cette distinction ne justifie par un recours au droit de la propriété, au même titre qu'un de ses organes. À cette première distinction importante, s'en ajoute une seconde, tout aussi importante, celle qui différencie la donnée et l'information. Comme nous l'avons mentionné dans l'introduction, la donnée est une information brute dépourvue de valeur d'usage. C'est le choix d'une valeur d'usage (clinique, scientifique, économique, etc.), suivant différents processus d'analyse et de traitement, qui la transformera en une information. Si la donnée génétique ne peut être assimilable à un bien, l'information, elle, pourrait l'être éventuellement. Mais ni le droit français, ni le droit de l'Union européenne ne se sont encore saisis expressément de cette question.

Une crainte est notamment nourrie par les revendications de brevets sur le génome [21]. En effet, la distinction entre donnée génétique et information, telle que défini précédemment, n'est absolument pas établie dans la loi. Or, si cela était le cas, le droit d'auteur pourrait être, dans ce cas, envisageable et applicable. Le passage d'une donnée en général en une information, si le niveau de transformation est suffisamment significatif (et que l'empreinte de la personnalité de l'auteur de la transformation est suffisamment identifiable; ce qui peut être difficilement concevable au regard de la complexité d'un tel processus), pourrait être considéré comme une création originale et relever du droit de la propriété intellectuelle. Mais attention, l'idée ici n'est pas de transformer la donnée génétique en une information génétique. Ceci ne serait évidemment pas suffisant pour qualifier cette information de création originale. Le processus de transformation, dans ce cas, serait de combiner une grande variété de données, dont génétiques, d'une personne, à celles de milliers ou millions d'autres personnes, afin de créer une ou des informations qui n'auraient plus rien à voir avec les données génétiques d'une seule personne initiale. Par ailleurs, ce processus garantit aussi une anonymisation et rend difficile toute ré-identification.

Cependant, le régime de protection de la propriété intellectuelle est très restrictif [21], notamment en raison de la nécessité de prouver le critère délicat de cette originalité [21]. D'où l'idée pour certains de s'appuyer plus largement sur le modèle du trust, ou celui de l'usufruit. Dans les deux cas, ils permettent aux «donneurs » de garder un contrôle sur l'usage de leurs données génétiques. Ils reçoivent, en revanche, une 
part sur la valorisation économique de leurs données effectuée par un tiers. Ceci remettrait alors en cause le principe de non-commercialité du corps humain [21]. C'est très probablement pour ces raisons que la France ne s'oriente pas, pour l'instant, dans ce sens.

\section{Perspectives et enjeux pour la France}

Actuellement, la France, et l'Europe en général, se concentrent sur un droit d'accès aux données génétiques. Celui-ci se fonderait sur une protection des bases données génétiques par un équilibre complexe entre droits d'auteurs, droits des utilisateurs et droit des contrats. Plus simplement, le droit français distingue la base de données protégée par le droit d'auteur si tant est qu'elle soit originale. Le contenu global de la base de données doit porter l'empreinte de la personnalité de l'auteur, protégé par un droit dit sui generis au bénéfice du producteur de la base de données; c'est-à-dire la personne physique ou morale qui a pris l'initiative et le risque d'investir substantiellement sur le plan financier, matériel ou humain pour mettre au point cette base de données ${ }^{7}$. Il convient d'ajouter à cela la protection éventuelle des données stricto sensu qui peuvent en elles-mêmes être originales et bénéficier de la protection du droit d'auteur. Cependant, les données génétiques brutes ne semblent pas constituer une «création originale » au sens du Code de la propriété intellectuelle en ce qu'elles ne constituent pas une œuvre de l'esprit ${ }^{8}$.

Pourtant, il est aujourd'hui indispensable que la France trouve une solution lui permettant d'optimiser la valorisation scientifique et économique de ces données génétiques et cela évidemment en toute transparence avec la société. Le Plan France Médecine Génomique 2025 [22], dont l'objectif principal est de favoriser un accès plus large au diagnostic génétique dans le cadre du soin, tout en permettant la recherche sur les échantillons biologiques ainsi obtenus et les données génétiques produites, donnera l'occasion d'appréhender ces questions. Mais derrière l'enjeu de la valorisation scientifique de ces données génétiques au travers du soin, suit inévitablement celui de leur valorisation économique au travers de la recherche, ce que le plan pourrait avoir l'opportunité de résoudre dans le cadre légal actuel.

Différents économistes réfléchissent, depuis longtemps, à la question de la valorisation économique des données en général et aux problèmes liés à la question de leur propriété. L'économiste français Jean Tirole a particulièrement bien posé le problème [23] : «l'entreprise qui dispose des données clients mérite-t-elle une rémunération liée à la possession de cette information? La réponse de bon sens [...] est que si cette collecte de données est le fruit d'une innovation ou d'un investissement massif, alors l'entreprise devrait pouvoir en profiter en les gardant pour elle-même. Si, en revanche, cette collecte est évidente et le coût de collection minime pour l'entreprise, les données

${ }^{7}$ Directive européenne $96 / 9 / C \varepsilon$, transposée par la loi du $1^{\text {er }}$ juillet 1998 et codifiée aux articles L341-1 et suivants du CPI. II sera observé que dans un rapport du 21 décembre 2015, la Commission européenne envisageait l'abrogation de la directive $96 / 9 / C \varepsilon$ au motif que ce dispositif nuirait au développement d'une économie de la donnée («data-driven ») européenne.

${ }^{8}$ Article L. 111-1 CPI. devraient appartenir à l'individu concerné ». Cependant, cette idée ne peut, pour l'instant, s'appliquer aux données génétiques, car le droit français rejette largement l'idée d'une appropriation, qui plus est «dans un but lucratif », quelle qu'elle soit.

Dans le cas des données génétiques, à moins que, finalement, la France décide de changer la loi, la solution pourrait finalement venir de cette idée de mettre en valeur la distinction entre donnée génétique et information. Car si la valorisation économique des données génétiques semble pour l'instant rendue difficile par le droit français et européen, la valorisation économique des informations tirées de l'analyse et du traitement est envisageable. L'intérêt alors de cette distinction entre donnée génétique et information est qu'elle permettrait une valorisation économique et scientifique de l'information, tout en maintenant le même niveau de protection sur les données génétiques. Les personnes pourraient garder un contrôle total sur l'usage de leurs données par l'intermédiaire d'un e-consentement dynamique [24] $(\rightarrow)$ et déci-

$(\rightarrow)$ Voir le Forum de H.C. Stoeklé et al., $m / s n^{\circ} 2$, février 2017 , page 188

der ou non de permettre à des tiers

d'utiliser «leurs » données génétiques et d'en tirer une information originale sans qu'il y ait besoin, en retour, de verser des «droits d'auteur». Suivant les différentes stratégies utilisées, il restera à choisir les modalités de partage et/ou d'échange.

Les nouveaux enjeux liés à la propriété et la valorisation des données génétiques devraient être posés et discutés en France, et cela à la fois dans l'intérêt de la prospérité de sa recherche et de sa médecine, mais aussi potentiellement de son économie. Parmi les choix, la France pourrait faire celui de faire évoluer la loi sur la protection des données génétiques ou d'établir une distinction franche entre donnée génétique et information. $\diamond$

\section{SUMMARY}

The ownership of genetic data: from data to information

In France, genetic data are not covered by property laws. They are considered to be equivalent to a part of the human body, to be protected rather than as something of economic value. However, in this highly competitive world, France must find solutions to increase the scientific and economic values of its genetic data. One possibility would be to define genetic data as raw information with no value for use. The choice of such a value of use (clinical, scientific, economic, etc.), following various key analysis and treatment processes will thus transform genetic data into useful information. In this case, 
and under certain conditions, intellectual property law could consider this information to be an original creation with an economic value, whilst maintaining current levels of protection for genetic data. France thus faces a choice between changing its laws concerning the protection of genetic data, bringing them into line with the North American approach, according to which data are a form of capital that everyone has the right to increase or sell, and making the distinction between genetic data and information clearer. $\diamond$

\section{REMERCIEMENTS}

Financement : ATIGE (Actions Thématiques Incitatives de Génopole).

\section{LIENS D'INTÉR}

Les auteurs déclarent n'avoir aucun lien d'intérêt concernant les données publiées dans cet article.

\section{RÉFÉRENCES}

1. Check Hayden E. Myriad Genetics embroiled in breast-cancer data fight-again. Nature 2016; $533: 449$.

2. Stoekle HC, Mamzer-Bruneel MF, Vogt G, Herve C. 23andMe: a new two-sided data-banking market model. BMC Med Ethics $2016 ; 17: 19$.

3. Spector-Bagdady K. "The Google of Healthcare": enabling the privatization of genetic bio/ databanking. Ann Epidemiol $2016 ; 26$ : 515-9.

4. Matloff $\varepsilon$, Caplan A. Direct to confusion: lessons learned from marketing BRCA testing. Am J Bioeth $2008 ; 8: 5-8$.

5. Orsi F, Moatti J. D‘un droit de propriété intellectuelle sur le vivant aux firmes de génomique : vers une marchandisation de la connaissance scientifique sur le génome humain. Economie Prévision $2001 ; 4: 123-38$.

6. Hervé C, Stoekle HC, Vogt G. Un marché aux données génétiques qui interroge. Le Monde 2016.

7. Stoekle HC. Médecine personnalisé et bioéthique: enjeux éthiques dans l'échange et le partage des données génétiques. Paris : L'Harmattan 2017 : 186 p.

8. Stenger T, Coutant A. Ces réseaux numériques dits sociaux. Paris : Hermès, 2011.
9. Proulx S, Heaton L, Kwok Choon M, Millette M. Paradoxical empowerment of produsers in the context of informational capitalism. New Rev Hypermedia Multimedia $2011 ; 17: 563-74$.

10. Armstrong M. Competition in two-sided markets. RAND J Economics 2006 ; $37: 668-91$.

11. Rochet JC, Tirole J. Platform competition in two-sided markets. J Eur Economic Association 2003 : 990-1029.

12. Lacour $\mathrm{S}$. Nouvelles technologies et patrimonialisation des données personnelles : un changement de paradigme? Personne et Patrimoine 2015 : 363-87.

13. Danos F. Propriété, possession et opposabilité. Economica $2007: 226$

14. Zenati-Castaing F, Revet T. Manuel de droit des personnes. Paris : PUF, 2006 : $240 \mathrm{p}$.

15. Caire A. Le corps gratuit : réflexions sur le principe de gratuité en matière d'utilisation de produites et éléments du corps humain. Rev Droit Sanitaire et Social $2015: 865$.

16. Le Corre F, Chassang G, Rial-Sebbag $\varepsilon$. Valorisation des éléments du corps humain : biobanques, propriété et commercialisation. Rev Générale Droit Médical $2017: 143$.

17. Titmuss R. The gift relationship: from human blood to social policy. London : LSE Books, 1970.

18. Waldby C, Mitchell R. Tissue economies: gifts, commodities, and bio-value in late capitalism. New York : Duke University Press 2006.

19. Noiville $C$, Supiot $\varepsilon$. Big pharma, big data et recherche génétique en santé. Rev Contrats $2015 ; 2: 352$.

20. Hayden $\varepsilon C$. Taking as giving: bioscience, exchange, and the politics of benefit-sharing. Social Studies Science 2007 ; 37 : 729-58.

21. Le Corre F, Chassang G, Rial-Sebbag $\varepsilon$. Valorisation des éléments du corps humain : biobanques, propriété et commercialisation. Rev Générale Droit Médical $2017: 155$.

22. Aviesan. France Médecine Génomique 2025. Paris : Aviesan, 2016.

23. Tirole J. Économie du bien commun. Paris : PUF, $2016: 534 \mathrm{p}$.

24. Stoekle HC, Deleuze JF, Vogt G, Herve C. Vers un consentement éclairé dynamique. Med Sci (Paris) $2017 ; 33: 188-92$.

\section{TIRÉS À PART}

G. Vogt

u'est-ce que la guérison? Des réponses, il y en a. De toutes sortes et de tout temps. Chacun y va de son savoir, religieux, scientifique, médical... Et de quoi est-on supposé guérir ? D'un symptôme, d'une douleur, d'une maladie, d'une répétition mortifère, d'un destin mélancolique ? Pour la psychanalyse, la guérison s'insère dans un système imaginaire et a, comme point de mire, un idéal. «La guérison, c’est une demande... »

\section{Qu'est-ce que} la guérison pour la psychanalyse?

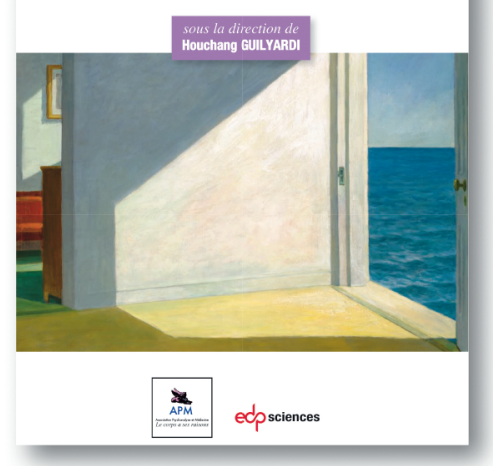
précise Lacan. Les auteurs nous invitent ici à découvrir, au-delà du semblant et à partir de la clinique, les liens entre guérison et vérité du sujet.

Comité éditorial de l'Association Psychanalyse et Médecine (APM): Martine Dombrosky, Sophie Dunoyer de Segonzac, Houchang Guilyardi, Josette Olier, Betty Testud

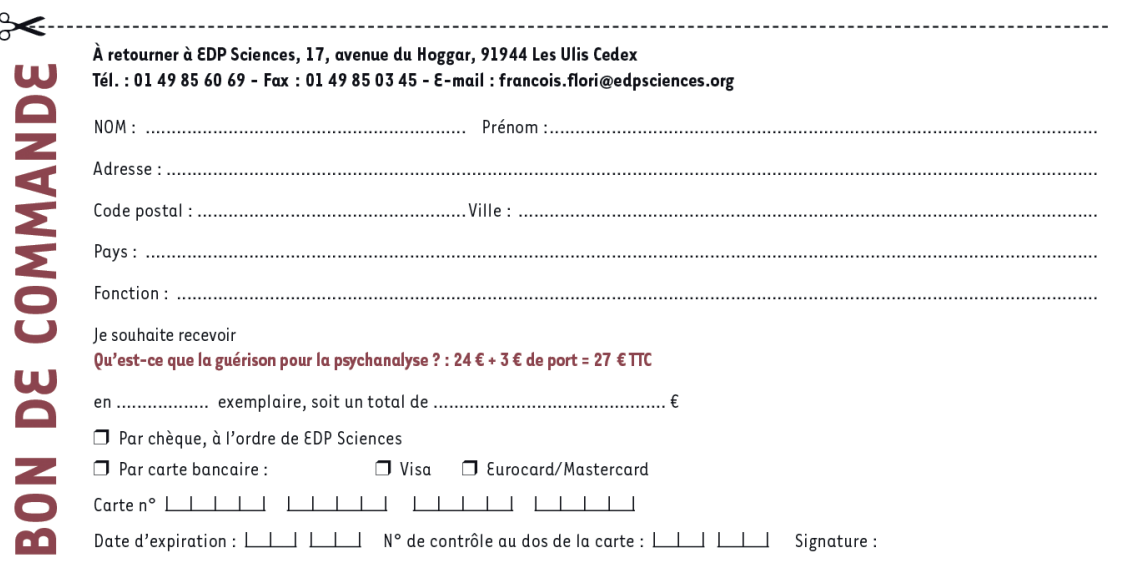

